\title{
SEPARATION AND CLASSIFICATION OF FETAL ECG SIGNAL BY ENHANCED BLIND SOURCE SEPARATION TECHNIQUE AND NEURAL NETWORK
}

\author{
Dr. Felix Joseph X, \\ Department of Electrical and Computer Engineering, \\ Bule Hora University, \\ Bule Hora, Ethiopia \\ felixjoseph75@gmail.com
}

\begin{abstract}
The separation of Fetal ElectroCardioGram (FECG) from mother's Abdominal ECG (AECG) signal is complicated and very important in medical diagnosis during pregnancy. In this study, the separation and classification of FECG signal from AECG signals is presented by a novel method. It uses MULTI-COMBI based Blind Source Separation (BSS) technique to separate the FECG signals. The separated FECG signals have different features, which are extracted using the morphological feature extraction method. These extracted features are used for classification by using Feed Forward Neural Network (FFNN). This classifier classifies the FECG into five different classes. The entire work is implemented in MATLAB. Results show that FFNN gives the classification accuracy of $77.1 \%$, sensitivity of $75.3 \%$, and specificity of $76.7 \%$.
\end{abstract}

Keywords: Blind source separation, fetal ECG, MULTI-COMBI, feed forward neural network.

\section{INTRODUCTION}

FECG separation is a very essential tool for heart rate monitoring of fetus. It is one of the important tests to diagnose the possible heart abnormalities in the fetus during pregnancy. Some of the possible diseases during pregnancy are cardiac arrhythmia, myocardial infarction, junction escape beat. The quality of diagnosis depends upon the quality of the signal. In normal case the ECG signals are mostly corrupted due to some noises such as power line interferences, EMG. In the FECG, the signal is additionally corrupted by the noises such as gestational age, skin impedance and position of skin electrodes [1].

There are five changes in fetal cardiac cycle. The electrical vector shift from the right atrium to left atrium has the electrical vector from which the wave is generated. Similarly $Q$ wave is generated when the electrical beat signal transferring through myocardial. $\mathrm{R}$ wave is the important peak value to find out the arrhythmia. The $\mathrm{S}$ wave is generated followed by $\mathrm{R}$ wave with negative changes. The depolarization of ventricles produces the $\mathrm{T}$ wave. This is the most important tool for the physician to find out the abnormalities in the heart activities of fetus. Commonly the FECG signals measured from two locations, such as chest and abdomen. The AECG signal contains the mother's ECG signal and FECG signal.

Various research works tells that different methods are presented to separate the FECG from AECG signal. Some of the different separation techniques are adaptive filtering [2], Wavelet transform [3], correlation techniques, and BSS techniques [4]. And some of the classification techniques are Neural Network [5], Fuzzy Logic [6], Naive Bay's Classification [7], and SVM [8].

This study proposes MULTI-COMBI method to separate FECG signal from the mixture of AECG signal. The different features are extracted by using 
morphological feature extraction method. The different classes of FECG are classified using FFNN method. The organization of this study is as follows: Section 2 describes the methods and materials used for FECG signal separation, feature extraction and classification. The results and discussion of the system are described in section 3. The last section concludes the FECG signal classification system.

\section{METHODS AND MATERIALS}

The process of separating FECG from mixed signal is done by using the technique BSS called MULTI-COMBI [9]. Efficient Fast Independent Component Analysis (EFICA) forms a cluster with components with Gaussian distributions. Weights-Adjusted Second Order Blind Identification (WASOBI) technique forms the cluster with components sharing similar correlation structures. MULTICOMBI technique uses this clustering technique in which both the algorithms EFICA and WASOBI are run on the set of un-separated sources $\hat{x}, \overrightarrow{I S R^{E F}}$ and $\widehat{I S R}^{W A}$ values are calculated. These processes are carried out until all clusters are singleton to one signal per cluster and hence the signals are separated optimally.

\section{A. MULTI COMBI Algorithm}

To initialize, take a stack of clusters which are denoted as ' $\mathrm{S}$ ', $\mathrm{S}=\{\mathrm{x}\}$ [10].

Step 1: Take a cluster from ' $\mathrm{S}$ ' and denote the cluster ' $\mathrm{z}$ ' and it is noted

that the cluster ' $\mathrm{z}$ ' will not be singleton.

Step 2: The EFICA and WASOBI are applied on the cluster $z$ and the ISR matrices values of $\widehat{S R}^{E F}$ and $\widehat{I S R}^{W A}$ are calculated by using the

formula

$$
\begin{gathered}
I S R_{k l}=\frac{1}{N} \frac{\gamma_{k}\left(\gamma_{l}+\tau_{l}^{2}\right)}{\tau_{l}^{2} \gamma_{k}+\tau_{k}^{2}\left(\gamma_{l}+\tau_{l}^{2}\right)} \\
I S R_{k l}=\frac{1}{N} \frac{\varphi_{k l}}{\varphi_{k l} \varphi_{l k}-1} \frac{\sigma_{k}^{2} R_{l}[0]}{\sigma_{l}^{2} R_{k}[0]}
\end{gathered}
$$

Step 3: Construct 'C' as a set with all possible clusters from $I \in\{1, \ldots \operatorname{dim}(z)\}$, i.e. if ' $\mathrm{z}$ ' consist of 3 signals, then the possible ' $\mathrm{C}$ ' will be $\mathrm{C}=\{\{1\},\{2\},\{3\},\{1,2\},\{1,3\},\{2,3\}\}$

Step 4: Compute $\widehat{I S R}^{E F}(I)$ and $\widehat{I S R}^{W A}(I)$ for $I \in C$

Step 5: Assume $E=\min _{I}$ and $W=\min _{I} \widehat{S R}^{W A}(I)$

Step 6: If $\mathrm{E}<\mathrm{W}$, then the set of best EFICA separated clusters can be picked,

$$
I_{1}=\arg \min _{I \in C} I \hat{S S R} R^{E F}(I)
$$

Continue for $\mathrm{k}=1,2 \ldots$ until the $\widehat{S R}^{E F}\left(I_{k+1}\right)>W$

The lowest ISR is picked by this procedure. EFICA separates the clusters one by one.

If $\mathrm{E}>\mathrm{W}$, extract $S_{1}, S_{2}, \ldots S_{M}$ from $S^{W A}$ using $\widehat{I S R}^{W A}$

Step 7: Update 'S' by substituting ' $z$ ' with $S_{1}, S_{2}, \ldots S_{M}$

Step 8: All the clusters in ' $\mathrm{S}$ ' are singleton, then stop. Otherwise return and starts from step 1 . 


\section{B. Morphological Based Feature Extraction Method}

After the separation of FECG signal, this signal will be given into the input of feature extraction. Morphological feature extraction method extracts the FECG signal features. The representations are described by these extracted features. The five deviations like $\mathrm{P}, \mathrm{Q}, \mathrm{R}, \mathrm{S}$ and $\mathrm{T}$ are present in the FECG signal are shown in Fig. 1. The peak detector is used to recognize the PQRST points in following ways.

$>$ The first upright (positive) wave labeled as $\mathrm{P}$ wave.

$>\mathrm{Q}$ is labeled as the first downward (negative) deflection.

$>\mathrm{R}$ wave is labeled as first upright (positive) next to $\mathrm{Q}$.

$>$ Negative deflection followed by the $\mathrm{R}$ wave is labeled as $\mathrm{S}$ wave

$>$ The first upright (positive) deflection after QRS is labeled as T.

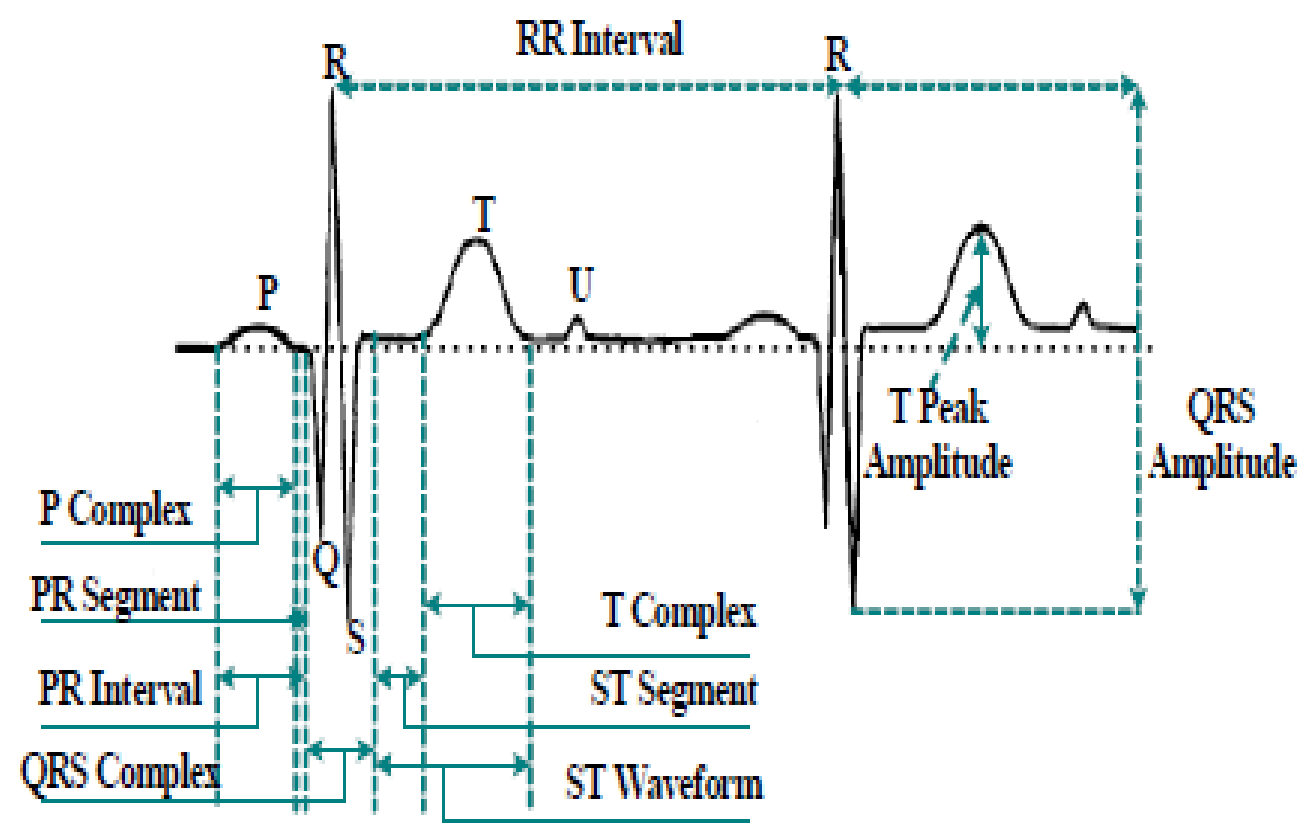

Fig. 1 Different features in FECG

From the five representations points the useful features are extracted by using morphological feature extraction method. There are three steps in morphological feature extraction method.

$>$ Find out the standard of PR, PT, ST, TT and QT interval

$>$ Take the number of R-peaks

$>$ Obtain the maximum value obtain in five representation peaks

- PR Interval

The value of PR interval is computing from the first modulation point in the FECG signal to the starting of QRS wave. The standard deviation and mean values are extracted from the given interval. Let the PR interval values are denoted by $\left[P_{R}\right]_{F}$, the mean value is given by, 


$$
\left[\mu_{P R}\right]_{F}=E\left[P_{R}\right]_{F}
$$

where $\left[\mu_{P R}\right]_{F}$ the mean value of fetal PR interval is, $E\left[P_{R}\right]_{F}$ is the expected time value of PR interval. Then the standard deviation value is calculated by the equation.

$$
\left[\left[\sigma_{P R}\right]_{F}=\sqrt{E\left[P_{R}^{2}\right]_{F}-\left[E\left[P_{R}\right]_{F}^{2}\right.}\right]
$$

where $\left[\sigma_{P R}\right]_{F}$ denotes the standard deviation of fetal interval PR are mean and standard deviation. The equation for mean is given by,

$$
\left[\mu_{P T}\right]_{F}=E\left[P_{T}\right]_{F}
$$

where $\left[\mu_{P T}\right]_{F}$ the mean value of fetal PT interval is, $\left[P_{T}\right]_{F}$ is the randomly obtained fetal PT interval, $E\left[P_{T}\right]_{F}$ is the expected period of fetal PT interval. The standard deviation of fetal PT interval is given as,

$$
\left[\left[\sigma_{P T}\right]_{F}=\sqrt{E\left[P_{T}^{2}\right]_{F}-\left[E\left[P_{T}\right]_{F}^{2}\right.}\right]
$$

where $\left[\sigma_{P T}\right]_{F}$ denotes the standard deviation of interval PT.

\section{- ST interval}

This interval is obtained in the interval starting from QRS complex wave which are starting at $\mathrm{T}$ wave. The standard deviation and mean values are calculated using ST interval. The mean value of fetal ST interval is given by

$$
\left[\mu_{S T}\right]_{F}=E\left[S_{T}\right]_{F}
$$

where $\left[\mu_{S T}\right]_{F}$ the mean value of ST is in interval, $\left[S_{T}\right]_{F}$ is randomly obtained from fetal ST interval, $E\left[S_{T}\right]_{F}$ is the period of fetal ST interval. The standard deviation of fetal ST interval is given by,

$$
\left[\left[\sigma_{S T}\right]_{F}=\sqrt{E\left[S_{T}^{2}\right]_{F}-\left[E\left[S_{T}\right]_{F}^{2}\right.}\right]
$$

\section{- QT interval}

The first negative deviation in FECG is called Q-wave. The interval is obtained between starting of $Q$ to the offset of $T$. Then mean value of fetal QT interval is calculated for the calculation of standard deviation of fetal QT interval. The mean value is defined by,

$$
\left[\mu_{Q T}\right]_{F}=E\left[Q_{T}\right]_{F}
$$

where $\left[\mu_{Q T}\right]_{F}$ the mean value of fetal QT interval is, $E\left[Q_{T}\right]_{F}$ is the expected time value of fetal QT interval. The standard deviation is given by,

$$
\left[\left[\sigma_{Q T}\right]_{F}=\sqrt{E\left[Q_{T}^{2}\right]_{F}-\left[E\left[Q_{T}\right]_{F}^{2}\right.}\right]
$$

where $\left[\sigma_{Q T}\right]_{F}$ is the standard deviation of fetal QT interval. 


\section{- Maximum peak value}

The maximum peak values are taken from the positive to negative side waves. After the identification of representation values waves, the maximum values $P_{\text {peak }}, Q_{\text {peak }}, R_{\text {peak }}, S_{\text {peak }}$ and $T_{\text {peak }}$ are calculated.

\section{- R-peak count}

The numbers of R-peak count $\left[R_{N}\right]_{F}$ in FECG signal is calculated after maximum peak values are calculated in representations wave. It is given by,

$$
\left[R_{N}\right]_{F}=N\left[R_{\text {peak }}\right]_{F}
$$

where $N\left[R_{\text {peak }}\right]_{F}$ is the number of R-peaks count.

\section{Classification of FECG Signal Using FFNN}

After the separation and feature extraction, the FECG is classified by using FFNN. In FFNN, the neurons are formed as layers namely input, middle and output layer, where, the input layer takes the input into the system, output layer produces the output, and the middle layer is also known as hidden layer because it do not have any contact with the external inputs. Each neuron is interconnected with each other which enhance the information. But there will not have any connection between the neurons in the same layer. The FFNN uses a supervised learning. In the case of back propagation learning algorithm (BPN), the input training vector for a training sample is presented every time. And output vector and the desired values are compared. The error can be calculated by using the squared difference of desired value and output vector as in the given equation,

$$
\text { Error }=(\text { desired value }- \text { output vector })^{2}
$$

The error shows the difference in distance from particular input and desired value. It leads in a desirable way to act in the network. Mapping function of inputs to output is learnt through the trained cases of input-output pairs. The output value is obtained by square and can be minimized by back propagation neural network. The three phases of training process are,

$>$ Send the signal pattern

$>$ Calculate the propagated error

> Update all the weights in the network

The features are extracted by morphological feature extraction method are given in input layer. Hidden layer optimizes using the weights and the five classes of FECG signals are grouped in the output layer. In this work, FFNN with three layers are used. The number of nodes in the input, hidden and output layer is 10 , 20 , and 5 . 


\section{RESULTS AND DISCUSSION}

The signals are taken from Physionet ATM bank [11]. The abdominal signals taken from the ATM bank are fed as an input of MULTI-COMBI algorithm. The output signals of mother's ECG signal, FECG signal and noise signal as shown in the Fig. 2.

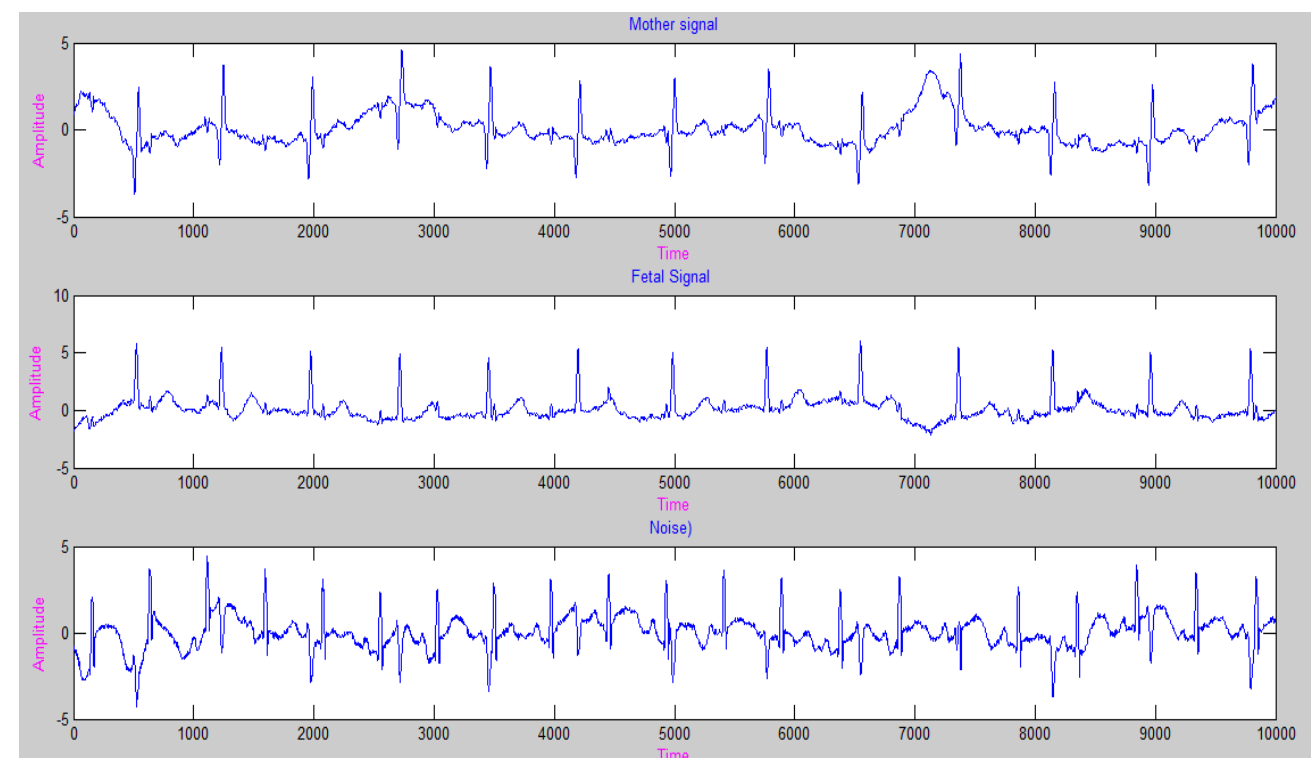

Fig. 2 Output waveforms of MULTI-COMBI algorithm

The FECG signal is taken for feature extraction using morphological feature extraction method. Figure 3 shows the minimum number of samples from the extracted FECG.

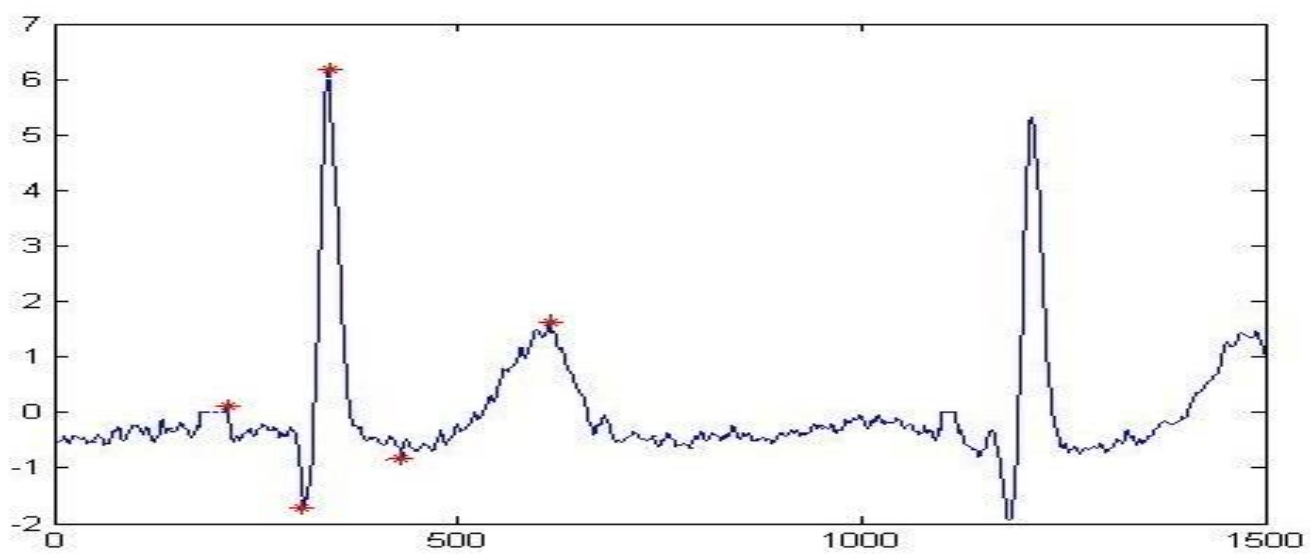

Fig. 3 Labeled representation for input signal 
The potency of MULTI-COMBI algorithm is proved by using PSNR and the average PSNR is 1.76 over all signals in the database. The effectiveness of the classifier is proved by using the parameters as sensitivity, specificity and accuracy. The number of true positives $\left(T_{p}\right)$ to the sum of true positive and false negative $\left(F_{n}\right)$ is called sensitivity. The sensitivity $\left(S_{t}\right)$ of feature extraction and classification is calculated by using the relation as,

$$
S_{t}=\frac{T_{p}}{T_{p}+F_{n}}
$$

The ratio of true negatives $\left(T_{n}\right)$ to the total value of true negative and false positive $\left(F_{p}\right)$ is called specificity. Specificity $\left(S_{p}\right)$ is calculated by the following equation

$$
S_{p}=\frac{T_{n}}{T_{n}+F_{p}}
$$

Accuracy $(A)$ of feature extraction and classifications are calculated by taking sum of total true values which are available in the population. It expressed as,

$$
A=\frac{T_{n}+T_{p}}{T_{n}+T_{p}+F_{n}+F_{p}}
$$

The extracted FECG signal is then classified into five different groups such as cardiac arrhythmia, myocardial infarction, functional escape beat, ventricular tachycardia, atrial flutter using FFNN classifier. The overall system performances (MULTI-COMBI + Morphological features + FFBN) are shown in Fig.4.

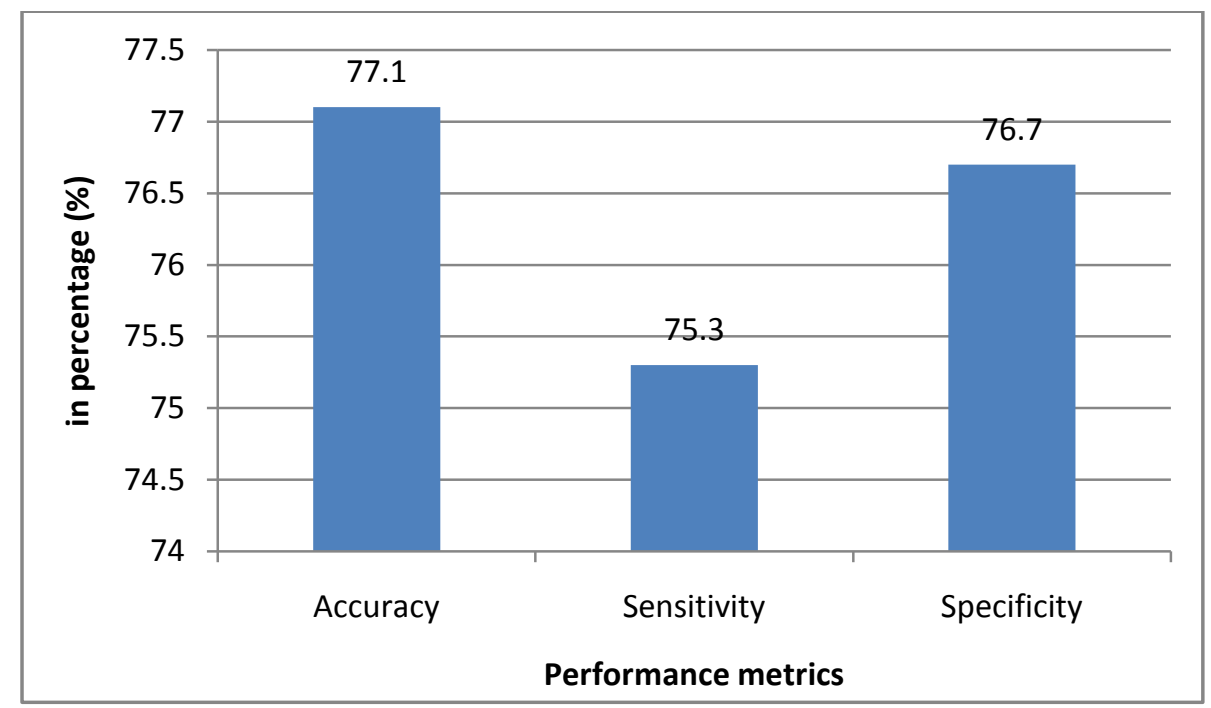

Fig. 4 Overall System Performance 


\section{CONCLUSION}

This study proposes the FECG signal separation using BSS algorithm. It also introduces feature extraction and classification methods for diseases classification. The separation BSS method gives better PSNR. The morphological feature extraction method produces ten features and FFNN classifier classify the FECG signal into five different classes such as cardiac arrhythmia, myocardial infarction, functional escape beat, ventricular tachycardia, atrial flutter. Experimental results on Physionet ATM bank database show the performance of the system which provides sensitivity of $75.3 \%$, specificity value of $76.7 \%$ and accuracy of $77.1 \%$.

\section{REFERENCES}

[1]. A. Kumar, L. Dewan, M. Singh, and H. Kurukshetra, "Real-time monitoring system for ECG signal using virtual instrumentation", IEEE Transactions on Biology and Biomedicine, vol. 3, 2006, pp. 638-643.

[2]. K. Ebrahim, B. Nooshin, and A. Karim, "A New GA-Based Adaptive filter for fetal ECG extraction", World Academy of Science, Engineering and Technology, 2011.

[3]. J. Reshu Bhoker, and P. Gawande, "Fetal ECG Extraction using Wavelet Transform", ITSI Trans. Electrical and Electronics Eng, Vol.1, No.4, 2013, pp. 2320-8945.

[4]. J.L. Camargo-Olivares, R. Martin-Clemente, S. Hornillo-Mellado, M.M. Elena, and I. Román, "The maternal abdominal ECG as input to MICA in the fetal ECG extraction problem", IEEE Signal Process. Lett. Vol. 18, 2011, pp. 161.

[5]. M. Korurek and B. Do gan, "ECG beat classification using " particle swarm optimization and radial basis function neural network," Expert Systems with Applications, Vol. 37, No. 12, 2010, pp. 7563-7569.

[6]. A. Sengur, "Wavelet transform and adaptive neuro-fuzzy inference system for color texture classification", Expert Systems with Applications, Vol. 34, No. 3, 2008, pp. 2120-2128.

[7]. S. Padmavathi, and E. Ramanujam, "Naïve Bayes Classifier for ECG abnormalities using Multivariate Maximal Time Series" Procedia Computer Science, Vol. 47, 2015, pp. $222-228$.

[8]. A. Kampouraki, G. Manis, and C. Nikou, "Heartbeat time series classification with support vector machines", IEEE Transactions on Information Technology in Biomedicine, Vol. 13, No. 4, 2008, pp. 512-518.

[9]. K. Prasanth, B. Paul, and A.A. Balakrishnan, "Fetal ECG Extraction Using Adaptive filters" International Journal of Advanced Research in Electrical, Electronics and Instrumentation Engineering, Vol. 2, No. 4, 2013, pp. 1483-1487.

[10]. J.F. Cardoso, "Multidimensional independent component analysis", IEEE International Conference on Acoustics, Speech and Signal Processing, 1998, pp. 1941-1944.

[11]. http://www.physionet.org 